\title{
Las industrias informativas: ¿tienen futuro? ${ }^{1}$
}

\section{Robert Picard²}

Recibido: 2014-06-10

Envío a pares: 2014-06-13
Aprobado por pares: 2014- 09-28

Aceptado: 2014-10-24

DOI: $10.5294 /$ pacla.2014.17.4.4

\section{Para citar este artículo / To reference this article / Para citar este artigo}

Picard, R. Diciembre de 2014. Las industrias informativas: ¿tienen futuro? Palabra Clave 17 (4), 1069-1096. DOI: 10.5294/pacla.2014.17.4.4

\section{Resumen}

La abundancia de información, la fragmentación y polarización de las audiencias, el desarrollo del portafolio de ofertas, los cambios en las formas de comunicar y el propio debilitamiento de las compañías mediáticas obligan a las industrias informativas a repensar sus modelos de negocio, ante el descenso ineludible de los ingresos provenientes del pago de los usuarios y la venta de publicidad. Algunos observadores confunden la difícil situación de los medios informativos con el periodismo. Pero estos no son sinónimos. El periodismo no es una forma de medio de comunicación; no es una plataforma de distribución; tampoco una industria o una compañía; ni un modelo de negocio, ni un trabajo. El periodismo es una actividad, un conjunto de prácticas mediante las cuales la información y el conocimiento son obtenidos, procesados y transmitidos. Las perspectivas económicas y empresariales resultan importantes para responder interrogantes sobre el estado actual y futuro de las industrias informativas, porque sus desafíos fundamentales involucran nuevas estrategias de financiamiento, costos y políticas públicas que permitan hacer frente a los retos ineludibles que se plantean en el siglo XXI.

1 Este texto se publica por primera vez en castellano en este número de la revista Palabra Clave. Traducido por Germán Arango-Forero. La versión original puede encontrarse en Picard, R. (2010). The Future of the News Industry (pp. 365-379). En Curran, J. (ed.). Media and Society. London: Bloomsbury Academic.

2 Director de investigación del Reuters Institute, Oxford University. Robert.picard@politics.ox.ac.uk. 


\section{Palabras clave}

Periodismo, industrias informativas, ingresos, financiación, crisis, modelos de negocio. (Fuente: Tesauro de la Unesco).

\section{The Information Industry: Does it Have a Future?}

\section{Abstract}

The wealth of information, the fragmentation and polarization of audiences, the development of the portfolio of offers, changes in the ways of communicating, and the weakening of the media companies forced the information industries to rethink their business models, in view of the unavoidable decline of revenues from the payments of users and the sale of advertising. Some observers mistake the plight of the media with journalism; but these are not synonymous. Journalism is not a form of media; it is not a distribution platform; it is also not an industry or a company, nor a business model, or a job. Journalism is an activity, a set of practices through which information and knowledge are obtained, processed and transmitted. The economic and business prospects are important to answer questions about the current and future state of the news industry, because its fundamental challenges do involve new financing strategies, costs, and public policies to deal with the inevitable challenges in the XXI century.

\section{Keywords}

Journalism, information industries, income, finance, crisis, business models. (Source: Unesco Thesaurus). 


\section{As indústrias informativas: têm futuro?}

\section{Resumo}

A abundância de informação, a fragmentação e a polarização do público, o desenvolvimento do portfólio de ofertas, as mudanças nas formas de comunicar e o próprio enfraquecimento das companhias mediáticas obrigam as indústrias informativas a repensar seus modelos de negócio, diante do inevitável declínio das receitas provenientes do pagamento de usuários e da venda de publicidade. Alguns observadores confundem a difícil situação dos meios informativos com o jornalismo. Mas estes não são sinônimos. $\mathrm{O}$ jornalismo não é uma forma de meio de comunicação; não é uma plataforma de distribuição; também não é uma indústria ou uma companhia; nem um modelo de negócio, nem um trabalho. O jornalismo é uma atividade, um conjunto de práticas através das quais a informação e o conhecimento são obtidos, processados e transmitidos. As perspectivas econômicas e empresariais são importantes para responder as perguntas sobre o atual e futuro estado das indústrias informativas, porque seus desafios fundamentais envolvem novas estratégias de financiamento, custos e políticas públicas para lidar com os inevitáveis desafios do século XXI.

\section{Palavras-chave}

Jornalismo, indústrias informativas, receitas, financiamento, crise, modelos de negócio. (Fonte: Tesauro da Unesco). 


\section{Introducción}

Las industrias informativas, entendidas como las actividades de los medios encaminadas a crear y difundir noticias e información, que han contribuido a la cohesión social y al sistema democrático durante casi tres siglos, atraviesan por un periodo de rápida transformación. Las estructuras y operaciones de este tipo de empresas dedicadas a informar han sido seriamente sometidas a prueba.

Tanto en Europa como en Norteamérica, los desafíos planteados a las industrias informativas han sido generados principalmente por cambios evidentes en las formas de comunicar — nuevas tecnologías que han permitido el incremento de los medios disponibles-, y una variedad de cambios sociales y de tendencias a nivel doméstico y global (Picard, 2004). Estos desafíos, ciertamente, han alterado la estructura corporativa de las empresas informativas que se consolidaron durante el siglo XX y han obligado a las compañías mediáticas a reestructurarse para reducir los costos de sus operaciones, provocando el despido de periodistas y de otro tipo de empleados, reduciendo como resultado la escala, el alcance, la recopilación y la difusión de cierto tipo de noticias (Reilly Center, 2008; Currah, 2009; Pickard, Stearns y Aaron, 2009).

La dificultad más evidente consiste en que el valor económico producido por este tipo de industria ha disminuido. Mientras los modelos de negocio de los medios comerciales se ven amenazados y han visto reducidos sus ingresos, las industrias informativas sin ánimo de lucro luchan por mantener el soporte financiero que garantizó su operación durante el siglo XX (Nissen, 2006; Almqvist y Thomas, 2008).

Los efectos de estos desafíos, en el caso de las empresas informativas, varían según las estructuras de los medios de comunicación. En algunos países, las noticias difundidas por televisión se han visto más afectadas que aquellas divulgadas en medios impresos; en otros lo ha sido la información divulgada por radio o por agencias de noticias; en ciertos países, la información de carácter nacional o metropolitana ha sufrido los efectos más 
notorios; mientras que en otros, el efecto se ha sentido más en noticias de carácter local o regional. El resultado general en las naciones más desarrolladas ha sido que el tiempo dedicado por las audiencias a las fuentes de información tradicionales ha disminuido. En general, los medios de los cuales la audiencia tradicionalmente solía recibir noticias e información, en los que los debates públicos eran moderados y las experiencias públicas eran generadas, están hoy recibiendo menos atención por parte de las audiencias.

Lo anterior no quiere decir que la necesidad de recibir información haya disminuido. Siempre se requerirán organizaciones mediáticas encargadas de producir y distribuir a diario contenidos de actualidad que garanticen la vigilancia de la sociedad y la exploración y el análisis en profundidad de asuntos y eventos de interés general. Lo que está cambiando, sin embargo, son las formas como las noticias están siendo financiadas, los medios por los cuales la información está siendo distribuida, la manera como las noticias están siendo consumidas y la propia sostenibilidad de las empresas informativas formalmente constituidas.

Algunos observadores confunden la difícil situación de los medios informativos con el periodismo. Pero estos no son sinónimos. El periodismo no es una forma de medio de comunicación; no es una plataforma de distribución; tampoco una industria o una compañía; ni un modelo de negocio ni un trabajo. El periodismo es una actividad, un conjunto de prácticas mediante las cuales la información y el conocimiento son obtenidos, procesados y transmitidos. Desde luego, estas prácticas están influenciadas por la naturaleza de los medios y las plataformas de distribución, así como por los acuerdos financieros que soportan la actividad periodística, pero uno no puede ser equiparado al otro. Este punto es importante porque el periodismo deberá adaptarse a las circunstancias cambiantes, pues su función en la sociedad sigue siendo significativa. El asunto que nos confronta hoy no es saber si el periodismo está llegando a su fin, sino saber cuáles son los modelos financieros y corporativos que crearán efectivas formas de producir información, y los mecanismos y las plataformas de distribución a través de los cuales las noticias podrán ser transportadas y entregadas en el futuro. 
Las perspectivas económicas y empresariales son importantes para responder interrogantes sobre el estado actual y futuro de las industrias informativas, porque sus desafíos fundamentales involucran estrategias de financiamiento, costos y políticas públicas. Estas aproximaciones fueron generalmente ignoradas durante la primera mitad de los estudios académicos sobre la comunicación, debido a que las investigaciones iniciales estuvieron concentradas en establecer los efectos individuales, sociales y culturales de los medios. Hoy, sin embargo, estudios relacionados con aspectos de orden estructural y operacional han aumentado más que nunca durante las últimas tres décadas, generando un cuerpo maduro y rico en literatura académica que busca explicar cómo aspectos económicos, regulatorios, gerenciales, administrativos y financieros ejercen presión, limitan actividades e influyen en las empresas mediáticas existentes determinando sus comportamientos (Albarran, 2005; Picard, 2005 a, b).

Estas perspectivas no se han centrado únicamente en el análisis de las relaciones empresa-mercado, sino también han abarcado rangos de estudio sobre la utilización de los recursos a nivel individual, de empresa, de la industria y la misma sociedad, y cómo los beneficios de esa utilización pueden ser maximizados. Las perspectivas de esta literatura nos ayudan a entender la importancia de estos cambios en la industria informativa, y pueden ser beneficiosas para decidir cómo responder a ellos, pues se deben encontrar soluciones para los asuntos que involucran tanto a consumidores como a la empresa, la industria y la sociedad como un todo.

\section{Cinco tendencias mediáticas decisivas}

Los cambios del entorno y la infraestructura de los medios están siendo impulsados por cinco tendencias mediáticas: abundancia, fragmentación y polarización, desarrollo del portafolio de ofertas, debilitamiento de las compañías mediáticas y un poderoso cambio en las comunicaciones.

La abundancia es el resultado de un crecimiento dramático en tipo y unidades de medios, ocurrido en la última parte del siglo pasado. El crecimiento de la oferta mediática ha excedido, de lejos, el crecimiento del consumo tanto en el sentido temporal como en el monetario. El desarrollo de 
la televisión por cable y satélite, además de Internet, creó nuevas formas de comunicación mediada que complementaron los contenidos informativos de la radio y la prensa escrita. Los cambios tecnológicos, combinados con un viraje ideológico sobre el rol del Estado en los diferentes mercados, han llevado a los entes reguladores a incrementar la competitividad entre los medios informativos de radio y teledifusión (Murdoch y Golding, 2001; Iosifidis, Steemers y Wheeler, 2005), y han cambiado el centro de las discusiones públicas hacia temas relacionados con el consumo y las necesidades de tipo individual, antes que en preocupaciones de orden colectivo. Los servicios públicos de radiodifusión ya no desempeñan los roles de claridad y protección como solían hacerlo en el pasado y deben competir con los nuevos actores.

La abundancia puede constatarse en el incremento, por tres, del promedio de páginas de los periódicos durante el siglo XX (Barnhurst y Nerone, 2001); el número de canales de televisión en Europa se multiplicó por tres entre 1995 y 2010 (European Audiovisual Observatory, 2009). Hoy hay cuatro veces más revistas de las que estaban disponibles hace 25 años; mil nuevos libros se publican en Europa a diario; 320 millones de horas de radio y 123 millones de horas de televisión se transmiten anualmente a nivel mundial; 1,5 millones de nuevas páginas se crean a diario en Internet, y nueva información crece a un promedio del 30 por ciento anual (University of California, 2004).

El efecto de esta abundancia es el ahogo de la audiencia en un mar de noticias, información y entretenimiento. Como consecuencia, las audiencias están fragmentando y polarizando su uso de los medios (Becker y Schönbach, 1999; Picard, 2002; Napoli, 2003); están repartiendo su uso de los medios a lo largo de más canales y más títulos, y esta fragmentación está produciendo extremos de uso y no uso (polarización). Existe una tendencia en Europa y Norteamérica entre los usuarios de medios, quienes concentran su uso en un periódico, en dos o tres revistas, en una o dos estaciones de radio y en tres o cuatro canales de televisión. Investigaciones sobre el uso doméstico de medios —en el caso de la televisión multicanal, por ejemplo- muestran que si se reciben veinte canales el promedio de 
audiencia en el hogar es de apenas cinco frecuencias; si la disponibilidad es de cincuenta canales, solamente doce son vistos en promedio. Y si la oferta asciende a cien canales, el promedio de consumo en el hogar apenas aumenta a dieciséis (Nielsen, 2003).

Los anunciantes publicitarios, que financian en gran medida la operación de los medios comerciales, responden a esta situación distribuyendo sus presupuestos entre más medios y pagando menos dinero por audiencias más pequeñas generadas por cada medio. Aún hoy, la televisión continúa recibiendo la participación de inversión publicitaria más grande, seguida por los medios impresos, la radio, la Internet, la publicidad exterior y el cine.

Un hecho especialmente preocupante tanto para los anunciantes como para las empresas mediáticas ha sido la progresiva tendencia de las audiencias a evadir los anuncios publicitarios (SIFO, 2008; Stuart, 2008). La creciente y abrumadora variedad de contenidos ha inundado a los públicos de mensajes comerciales, provocando consigo una gran fatiga de publicidad y de mensajes comerciales. Este se ha convertido en uno de los principales incentivos para adquirir programas de cómputo y sistemas que permiten evadir secciones comerciales tanto por televisión como en Internet. En Estados Unidos, por ejemplo, 54\% de los usuarios de Internet cuentan con bloqueadores de mensajes no deseados, $60 \%$ de quienes poseen grabadores de video digitales los utilizan para evadir o para borrar comerciales y cerca de 60 millones de hogares están inscritos en las listas de "no llamar" en el sistema de telemercadeo. El nivel de evasión se reduce en la medida en que aumenta la edad de la audiencia, el rango comprendido entre los 15 y los 24 años presenta los mayores índices de rechazo, un grupo primordial para los intereses de los anunciantes de bienes y servicios. La evasión registra los niveles más bajos en el caso de los medios de carácter personal, excepto en los correos no deseados, pues los mensajes tienden a ser más del tipo de anuncios de índole personal y un mercadeo más del estilo de relación con el consumidor. El efecto promedio de la evasión, en todo caso, es el de hacer la publicidad tradicional menos atractiva para los anunciantes e incrementar la búsqueda de oportunidades para alcanzar consumidores reales y potenciales. 
Las compañías mediáticas han respondido a esta situación con un portafolio de estrategias, debido a que el declive de la rentabilidad promedio de cada empresa hace cada vez más problemática la propiedad de un solo medio. La mayoría de compañías ofrece hoy múltiples contenidos en un esfuerzo por reducir los riegos y obtener economías de escala y de alcance limitados (Picard, $2005 \mathrm{a}, \mathrm{b}$ ). Hacen esto porque los portafolios pueden incrementar el retorno de la inversión a través de una mayor eficiencia económica en sus operaciones y producir ahorros conjuntos de costos. El desarrollo de estos portafolios puede ser ejemplificado por la editorial francesa del magazín Lagardére (Hachette Filipachi), que publica más de cuarenta títulos como Elle, Paris Match, Car \& Driver, Women's Day y Road and Track, y la editorial británica AMAP, que publica cerca de seis docenas de magazines profesionales incluyendo a Broadcast, Nursing Times, Architectural Review y New Civil Engineer.

A pesar del desarrollo de estos portafolios, y de la importancia de las comunicaciones en el mundo contemporáneo, la fortaleza de las compañías mediáticas sigue erosionándose. Por primera vez en medio siglo, compañías de medios que no se dedican a la producción de contenidos figuran entre las cien empresas más importantes en Estados Unidos o en las grandes naciones europeas. Esto ocurre porque el alcance de las companías mediáticas está disminuyendo a pesar de que han crecido en promedio. La corporación Disney, por ejemplo, suele ser utilizada como modelo de concentración mediática en Estados Unidos y a nivel global. En los años setenta, el canal de televisión $\mathrm{ABC}$ (que para entonces tenía una relación financiera con Disney pero no era totalmente propiedad suya) servía en promedio a entre el 25 y el $30 \%$ de la audiencia de televisión norteamericana diariamente. Hoy, todos sus canales de televisión abierta y por cable combinados (ABC, Disney Channel, ABC Family, ESPN, Soap Net, más los canales en los cuales cuenta con participación accionaria como A\&E, Lifetime y E!) alcanzan en promedio menos del $20 \%$ de la audiencia en Estados Unidos. Por tanto, mientras la compañía cuenta con más canales y ha crecido en tamaño, su nivel de influencia sobre la audiencia realmente ha disminuido. 
Aunque los conglomerados de medios más fuertes generan ingresos entre 25 y 50 billones de dólares anualmente, son relativamente empresas medianas en comparación con el promedio de las grandes compañías. Time Warner, el grupo mediático más grande incluido por la revista Forbes en la lista de las 500 empresas a nivel mundial, se ubicó en el puesto 150 en el ranking de ingresos. La holandesa Shell es diez veces más grande que Time Warner, mientras que Wal-Mart es 16 veces más grande que el gigante mediático alemán Bertelsmann.

El tamaño de las grandes compañías mediáticas en términos de personal, activos e ingresos, lleva a algunos analistas a concluir que están incrementando su escala y su alcance para consolidar grandes sinergias y obtener beneficios financieros. Investigaciones han demostrado, sin embargo, que tales sinergias y beneficios son difíciles de conseguir y que cerca de tres cuartas partes de todas las adquisiciones fallan a la hora de producir beneficios para las compañías. En la última década, en Estados Unidos las compañías mediáticas líderes enajenaron cinco veces más unidades de negocio de las que adquirieron, debido a que muchas de las adquisiciones realizadas en los años noventa resultaron perjudiciales para el valor y el desempeño de las compañías.

Debido a sus condiciones más débiles, a las grandes compañías mediáticas a nivel global les preocupa que puedan convertirse en objetivos de adquisición y tratan de implementar mecanismos de protección. Muchas compañías mediáticas actualmente lidian con sus máximos inversionistas acerca de las estrategias implementadas y los rendimientos obtenidos.

Subyacente a todas estas transformaciones está también un cambio de poder en las comunicaciones. En el pasado, el espacio mediático estaba bajo el control de las compañías mediáticas, pero hoy ese espacio está cada vez más controlado por el consumidor. Ha cambiado de una demanda impulsada por la oferta, a una oferta impulsada por la demanda. Lo podemos ver en la financiación de nuevas iniciativas en el mercado de la televisión por cable y satélite, en la radio satelital, en las descargas de audio y video por Internet, en la televisión digital en la mayoría de naciones, y en los medios móviles, cuyos modelos de negocio están basados en el pago por parte de 
los consumidores. En promedio mundial, por cada euro que es invertido en los medios por los anunciantes publicitarios, los consumidores ahora invierten tres euros. La cifra correspondiente en el caso de Estados Unidos es de un euro por siete y en el Reino Unido es de un euro por 5 (PriceWaterhouseCoopers, 2005).

Los mayores anunciantes están hoy constriñendo sus presupuestos publicitarios porque los encuentran menos efectivos que en el pasado en términos de ventas y retorno de la inversión. Hoy la publicidad en medios alcanza cerca de un tercio del presupuesto total destinado a gastos de mercadeo por parte de las grandes compañías, las cuales han orientado su estrategia hacia labores de mercadeo personalizado, directo, patrocinios, promoción con otras marcas, entre otros.

El cambio de poder en las comunicaciones también resulta evidente en la producción de contenido, donde las tecnologías permiten una creación de contenidos más fácil para el consumidor. El desarrollo de las tecnologías de la información y la comunicación ha estado acompañado por la implementación de programas de cómputo para la creación de audio, video, páginas de Internet, sitios personales y blogs (Küng, Picard y Towse, 2008). En el pasado, la creación de este tipo de material requería habilidades profesionales significativas, pero hoy los programas de cómputo incorporan esas habilidades y las hacen accesibles a un rango mayor de personas para producir y diseminar contenidos. Esta creación ha sido apoyada por tecnologías que promueven compartir contenidos entre pares, construir redes sociales, disfrutar juegos colaborativos y realizar actividades comunales. Todas estas nuevas actividades comunicativas y mediáticas, que fueron originalmente creadas para su uso en computadores o en lugares fijos, están cambiando más y más hacia usos portátiles gracias a los teléfonos móviles, e incrementalmente les están permitiendo a los usuarios interactuar a voluntad durante el transcurso de sus días.

\section{Efectos individuales y sociales}

Los roles de los medios en la sociedad y las relaciones tradicionales de la misma también están cambiando (Castells, 2001; Bakardjieva, 2005; van 
Djik, 2005; Fuchs, 2007) debido a la forma como los medios están siendo utilizados por las audiencias, los usuarios, los consumidores y los ciudadanos. Estas conceptualizaciones sobre los individuos en el ambiente mediático actual resultan significativas, pues indican el tipo de relación que tiene lugar entre las personas y los contenidos de los medios de comunicación. Las relaciones no son mutuamente excluyentes, desde luego, y los individuos desempeñan distintos roles en diferentes momentos dependiendo de cuándo, dónde y cómo emplean los medios.

El concepto de audiencia proviene de una época con un flujo de comunicación simple, es decir, en una sola vía, en la cual el público desempeñaba un rol eminentemente pasivo y receptivo. La audiencia ha sido siempre un concepto imperfecto y abstracto, basado en la sumatoria de aquellos que escuchan, ven o leen medios, y esto generalmente representa a aquellas que responden a características de su totalidad y existencia, pero sin opciones, preferencias o efectos. El concepto de usuarios surge de individuos más activos en el empleo de medios y de tecnologías para satisfacer sus gustos y necesidades. En este concepto relacional, los individuos escogen los medios y los contenidos que desean, interactuando con ellos y controlándolos de los modos que ellos prefieren. El concepto del consumidor reconoce una relación basada en el mercado, en la cual los individuos intercambian dinero a cambio de bienes y servicios proveídos por los medios, y hace énfasis en el grado de influencia que tienen los individuos en esta relación de intercambio. Finalmente, el concepto de ciudadano enfatiza el rol social de los individuos en la sociedad y su uso de los medios para desempeñar dichos roles.

Muchas de las dificultades que los medios informativos afrontan hoy ocurren precisamente por el cambio de rol de los individuos, quienes han pasado de tener un comportamiento como audiencia a sostener una relación del tipo usuario y consumidor.

Esto hace más difícil la financiación de las empresas informativas, pues la atención pública a las noticias y a los contenidos es limitada en comparación con la atención prestada a los contenidos relacionados con entretenimiento. En el ámbito empresarial, las labores informativas han sido vistas siempre como centros de costos, cuyos gastos son absorbidos por los ingre- 
sos generales generados por las empresas mediáticas comerciales. La relación del ciudadano no es tan fuerte como aquella de la audiencia, el usuario y el consumidor, y esto involucra principalmente a observadores sociales y a activistas políticos, porque tiene una implicación significativa en la manera como los individuos desempeñan sus roles como ciudadanos.

Debido a las múltiples opciones relacionadas con los contenidos mediáticos, los individuos dedican ahora tiempo considerable a usos alternativos de medios interactivos. Esta variedad creciente en el uso de los medios está creando desafíos significativos para los medios de información y entretenimiento formalmente establecidos, pues ellos develan limitaciones y defectos de los productos, y debilidades en la orientación del usuario y consumidor de contenidos informativos. La presentación de noticias nuevas y de productos informativos elaborados o distribuidos por firmas recién llegadas está destruyendo las economías a escala que previamente existían y están provocando deficiencias organizacionales y estructuras de costos insostenibles entre las empresas informativas tradicionalmente establecidas.

En este ambiente, los cambios no son una opción posible para los directivos y gerentes de medios, son una obligación. El tiempo y la naturaleza de los cambios afectan los medios informativos de distinta manera. Las audiencias de medios audiovisuales se ven más afectadas que las de medios impresos, pero las condiciones financieras de los periódicos se ven más perjudicadas, pues tienen el porcentaje más alto de costos operacionales no relacionados con contenidos. En países con una estructura de medios de orden regional o local, por ejemplo Estados Unidos o Francia, los medios que operan a nivel nacional y en grandes áreas metropolitanas se ven más afectados que empresas mediáticas más pequeñas de orden local, debido a que más competidores proveen noticias e información de orden metropolitano, nacional e internacional. En países donde los medios dominantes operan a nivel nacional, como el Reino Unido, por ejemplo, los medios regionales o locales muestran mayores debilidades.

Las decisiones personales e independientes hechas por los individuos como miembros de la audiencia, como usuarios y como consumido- 
res están teniendo efectos colectivos (Picard, 2002). Estos efectos pueden verse en grupos de audiencias con formas particulares de uso de los medios de comunicación. Algunos son usuarios más habituales de medios impresos; otros favorecen el uso de medios audiovisuales; otros prefieren la radio, mientras algunos ponen mayor énfasis en el uso de Internet y de comunicaciones móviles. Algunos son más pasivos en sus usos, mientras que otros resultan más activos.

Las audiencias pasivas y los consumidores prefieren leer periódicos, revistas y libros, además de ver televisión abierta. Individuos atraídos por el video muestran preferencia por la televisión vía cable o satélite, contenidos en DVD y descargados por Internet, e incrementalmente se están alejando de la televisión abierta. Personas atraídas por el audio prefieren escuchar radio, grabaciones, dispositivos con formatos en MP3 y ver videos musicales. Todas las audiencias pasivas desempeñan el rol de receptores en la comunicación y tienen poca interacción con el medio o con otra persona a través del medio escogido.

Aquellos que activamente consumen contenidos mediáticos pueden ser conceptualizados como conversadores (los que prioritariamente utilizan voz, mensajes de texto y correos electrónicos para sostener conversaciones), creadores (los que utilizan texto, audio y videos a fin de crear mensajes para grupos más grandes de personas) y absorbentes (los que gastan tiempo significativo buscando información, jugando, etc.). La mayoría de las personas combinan roles pasivos con activos en el consumo, pero la mayor parte del tiempo utilizado en los medios tiende a ser de uso pasivo.

Las personas difieren ampliamente, también, en términos de consumo de información y noticias. Los grandes consumidores tienden a ser individuos activos política, social y económicamente, quienes buscan amplitud y profundidad de noticias e información desde distintas fuentes y plataformas. Ellos tienden a representar únicamente entre el 15 y el $20 \%$ de la población en la mayoría de países. En promedio, los consumidores de noticias e información quieren noticias generales e información sobre tópicos que afectan sus vidas e intereses de forma directa e inmediata. Tienden a sen- 
tirse satisfechos con información rápida y simple, con contenidos con una visión general de los hechos proporcionados por periódicos y noticieros tradicionales, así como por titulares e historias centrales en portales de Internet. Este grupo representa entre el 60 y 70 \% de la población. El resto, entre 15 y $20 \%$ de la población, son consumidores de pocas noticias e información, quienes tienden a estar conectados marginalmente con la sociedad y cuentan con ingresos económicos y niveles de educación más bajos. Ellos tienden a evitar contenidos informativos y prefieren enfocar su uso de los medios en contenidos de entretenimiento de carácter pasivo.

Debido a que gran parte del público no es buscadora activa de información - excepto los periodistas y esa pequeña parte de la población que actúa sobre las noticias-, la mayoría de proveedores comerciales de información y noticias han buscado servir tradicionalmente al grupo más grande, y muy pocos medios se han convertido en proveedores de información para los usuarios más activos. Los periódicos y los noticieros han sido cargados con contenidos hechos para atraer a la gran audiencia. Enormes cantidades de espacio y de tiempo se han dedicado al entretenimiento, el deporte y los estilos de vida (farándula) con el objetivo de ser leídos, escuchados o vistos. Actualmente, en la mayoría de los periódicos el contenido que puede ser considerado como verdadera información y noticia cuenta con menos del $20 \%$ del total, pero esta estrategia ya no está funcionando porque mucha de esa información ligera ahora está disponible de manera más fácil y barata para adquirir, usar y obtener en muchos otros lugares.

Hoy, la calidad de los medios impresos que han estado destinados a usuarios de alto nivel está siendo fuertemente afectada por la disminución de los recursos financieros. El consumo de información y noticias por parte de los usuarios promedio ha disminuido, con la consecuente reducción del mercado masivo de noticias e información para medios impresos y televisivos que fue creado durante los siglos XIX y XX, privando a las empresas mediáticas de una buena parte de los ingresos que generaba.

En el Reino Unido, por ejemplo, la circulación de los diarios era de 15,8 millones en 1960 y de 17,5 millones en 2008. Aunque el número au- 
mentó, la tasa de crecimiento fue la mitad en comparación con el crecimiento de la población durante el mismo periodo, por tanto, el alcance de la publicación ha disminuido. En Estados Unidos, las grandes cadenas de televisión ( $\mathrm{ABC}, \mathrm{CBS}$ y $\mathrm{NBC}$ ) tenían durante la noche un alcance promedio de 52 millones de televidentes en 1980, pero ese número se redujo de manera constante a solo 29 millones en 2008. La circulación de los diarios era de 53,8 millones en 1950 y pasó a 48,6 millones en 2008. En comparación con la tasa de crecimiento de la población, el número de copias por persona disminuyó un $50 \%$.

Estas tendencias están obligando a las organizaciones de noticias a tratar de incrementar los pagos de los grandes consumidores para poder cubrir los costos, y a proveer noticias e información con tarifas más bajas, incluso gratis, a los usuarios promedio, convirtiendo las noticias en un producto básico sobre una variedad de plataformas. Las dos estrategias crean desafíos financieros debido a que hay modelos de negocio limitados disponibles para financiar la operación de los medios.

En el modelo de costo al consumidor, este paga por el consumo una vez o por una suscripción temporal al contenido del medio. Algunos medios fijan un precio bajo que permite un uso básico, mientras que un precio más alto permite un uso más extendido del contenido, una forma de mejorar la venta. El pago de publicidad implica para los anunciantes acceder a unas audiencias mediante la compra de espacio o de tiempo en los medios, y en el mundo de Internet la posible interacción con potenciales compradores. En estas nuevas formas de pago, los anunciantes pagan significativamente por formas interactivas como poder hacer click en enlaces y por contactos comerciales generados, pero no están dispuestos a pagar mucho por la simple exposición de los mensajes a las audiencias como en el caso de los medios tradicionales.

Una tercera forma de ingreso es el patrocinio, mediante el cual ciertas compañías pagan por ser relacionadas con determinados productos y servicios mediáticos. Esta modalidad frecuentemente se representa en un mensaje del tipo "con el patrocinio de... ofrecido por... gracias a ...", o a través 
de una exposición visual de la marca. Una cuarta forma de ingreso proviene de licenciar contenidos para ser utilizados por otras compañías a cambio de un pago. Finalmente, existe el financiamiento estatal o el impuesto por el Estado (como en el caso de los derechos de licencia o de funcionamiento para financiar el servicio público de radio y televisión).

Recientemente, ha surgido el interés por apoyar las operaciones de las organizaciones informativas sin ánimo de lucro, pero esto no constituye una panacea para los desafíos que afronta el periodismo, pues estas empresas, en todo caso, requieren de un capital y de unos ingresos para poder operar, y porque muchas de estas organizaciones informativas sin propósitos comerciales son relativamente mediocres. Incluso, algunos sistemas informativos públicos no han sido inmunes a los retos económicos contemporáneos.

Cómo crear valor y ofrecer contenido informativo único, no disponible fácilmente en otros lugares, se ha convertido en un elemento crítico para el sostenimiento de las empresas informativas (Picard, 2006). Aunque gran parte del periodismo produce valor social, no resulta suficiente con pagar los salarios a los periodistas y con mantener solventes a las empresas informativas. Hoy, tanto los unos como las otras están siendo forzados a confrontar asuntos sobre cómo crear valor económico y garantizar fuentes de ingresos.

Durante varias generaciones, directores y gerentes no tuvieron que preocuparse mucho por estos asuntos, pues las estructuras sociales, técnicas y económicas protegían a las empresas informativas. Pero las tendencias que están orientando el cambio en el negocio de las noticias han empujado a la industria más allá de un único periodo, financieramente dorado en su historia. Directivos y trabajadores de la industria informativa tienen larga experiencia sobre un periodo en el cual la competición limitada, los generosos derechos otorgados por las licencias y el gran crecimiento de los ingresos por concepto de publicidad proveían abundantes recursos que permitían un extraordinario crecimiento en el tamaño de las empresas informativas, particularmente entre 1970 y 1990 . Hoy, la riqueza que produjo 
semejante crecimiento está siendo despojada del negocio de las noticias. Esto se hace evidente en el hecho de que en Estados Unidos y en el Reino Unido, la inversión de publicidad en los periódicos ha experimentado en promedio una tasa de crecimiento de entre 2 y $0,5 \%$ respectivamente desde el año 2000 hasta 2008 (World Press Trends, anual).

Entonces, ¿qué deben hacer las empresas informativas? Para sobrevivir y prosperar deben proveer noticias e información mejores y diferentes que las ofrecidas por la competencia, y ofrecer noticias e información para aquellos usuarios que sean capaces de valorarlas. Para lograrlo, las empresas informativas deberán cambiar las formas como utilizan el personal y asignan los recursos financieros, y tendrán que modificar además sus fuentes actuales de ingresos. Para alcanzar esto último, con el tiempo deberán transferir los costos a los usuarios de productos informativos de calidad, aumentando así su precio de adquisición. Estas empresas solo serán capaces de hacerlo si se concentran en estrategias efectivas para el mejoramiento de la calidad de los contenidos.

Los medios informativos, tanto si sirven a los segmentos de usuarios exigentes como a los considerados promedio, deben desarrollar múltiples productos para distintas plataformas. Los usuarios exigentes desean este tipo de productos, de modo que puedan adaptarse a esta clase de desarrollos y obtener información en cualquier momento y lugar. Los medios que sirven a los usuarios promedio necesitan reutilizar el material que producen y crear múltiples puntos de contacto mediante los cuales puedan nutrir de mensajes publicitarios a sus usuarios.

En el nuevo entorno, está claro que existen roles y funciones para el uso de blogs y de redes sociales para el acopio y la difusión de noticias e información, así como foros públicos para la discusión. Las tecnologías contemporáneas de comunicación soportan estos medios de pensamiento colectivo. Cuando no están generando una discusión cara a cara, los blogs y las redes sociales asistidas tecnológicamente se encuentran incrementando las oportunidades de los individuos para transmitir sus ideas y opiniones, para informarse mutuamente, para responder y comprometerse con conversaciones de formas que solían estar limitadas por los medios de 
comunicación masivos tradicionales. En la actualidad, las tecnologías comienzan a permitir metaanálisis efectivos sobre rumores, blogs y redes sociales que reúnen temas y un sentido generalizado de las opiniones que se expresan. Estas tecnologías de la información, por tanto, permiten agregar millones de puntos de vista y de opiniones de formas que anteriormente no eran posibles.

A dónde nos van a conducir estas tecnologías, aún no está tan claro. Sin embargo, el compromiso y la participación en línea de millones de personas resultan mucho más convenientes para la sociedad que la privación de derechos que la sociedad de masas previamente alentó. Las organizaciones mediáticas tendrán que luchar con las formas como esa contemplación colectiva altera los roles y las funciones de los editorialistas, líderes de opinión y columnistas. Ellos tendrán que comprometerse cada vez más con el público y revisar sus roles como creadores de temas de opinión, no simplemente diciéndole a la gente lo que piensan u opinan sobre alguna materia.

Estas oportunidades ofrecidas por las tecnologías de la comunicación están demostrando gran utilidad en la recopilación de información, particularmente para las noticias de última hora y para obtener información del público cuando los periodistas no están presentes en el cubrimiento de los eventos. El significado de esto fue crudamente ilustrado por las fotos, los videos y la información provenientes de Irán en la primavera y el verano de 2009 durante las protestas por las controvertidas elecciones presidenciales.

Los contenidos generados por los usuarios también permiten el cubrimiento de algunos eventos y temas de interés, cuando las limitaciones presupuestales de los medios no permiten realizar un cubrimiento profesional. De esta manera, el público se convierte cada vez más en un proveedor de información sobre comunidades y organizaciones, eventos deportivos a nivel aficionado y otro tipo de asuntos que generalmente no son cubiertos por los periodistas.

Uno de los grandes efectos de los medios de búsqueda y de las redes sociales ha sido el de convertirse en plataformas para la redistribución de 
contenidos producidos profesionalmente. Esta redistribución puede tanto beneficiar como perjudicar a las empresas informativas, dependiendo de si tales plataformas conducen a lectores, oyentes y televidentes a la fuente original de información, o si la transmiten sin llevar a los individuos a dicha fuente, la cual se financia de la inversión publicitaria basada en el número de usuarios que consumen dicho material.

Las empresas informativas aún tratan de encontrar la creación de valor más eficiente de las actualizaciones de los blogs, los podcasts, los mensajes de texto, los sistemas de mensaje multimedia, los tuits y otras redes sociales. En una época en la que disminuyen los ingresos y el personal, serios cuestionamientos deben hacerse sobre los beneficios de las nuevas tecnologías para el periodismo y para sus modelos de negocio. ¿Es cada uno de ellos igualmente útil? ¿Cuáles son los costos reales en tiempo laboral y en gastos de operación de las diversas plataformas? ¿Qué se consigue realmente generando presencia allí por parte de las empresas informativas? ¿Todas las organizaciones informativas tienen que estar activas en todas las plataformas? ¿Cómo puede una empresa informativa obtener beneficios óptimos a través de estas plataformas? ¿Cómo podrán generar o promover ingresos por otros medios? Las respuestas y las decisiones tomadas varían según el tipo de empresa, sus circunstancias y oportunidades.

Millones de personas emplean nuevas tecnologías. Sin embargo, incluso en esta época de exploración y experimentación, es claro que los usuarios de estas herramientas digitales reaccionan a ellas de diferentes maneras. Algunos las encuentran muy útiles y satisfactorias. Otros no les encuentran su valor y las consideran decepcionantes. No todo el mundo quiere estar, o estará igual de conectado, comunicando o compartiendo sus opiniones y los detalles de sus vidas. Algunos, incluso, encuentran las tecnologías de la comunicación más gratificantes en el campo de los negocios. Otros hacen énfasis en los beneficios de índole personal. Por consiguiente, muchas de estas tecnologías sirven hoy a una fracción de toda la audiencia digital, en el mejor de los casos, entre el 5 y el $20 \%$. Esto también debe tenerse en cuenta y las empresas de comunicación deben evaluar de manera realista las oportunidades potenciales que buscan crear. 
Todavía es temprano para definir el uso de las tecnologías por parte de las empresas informativas. No obstante, ya podemos encontrar algunos indicadores sobre la efectividad de estas tecnologías de mensajería instantánea interactivas y sociales. Es claro que tienden a beneficiar más a organizaciones de noticias de orden nacional y metropolitano, de lo que resultan para empresas locales más pequeñas; esto ocurre debido a que ofrecen ventajas competitivas para convertir la marca en omnipresente en medio de las numerosas y competitivas fuentes de noticias e información. Cuando su uso está mucho más dirigido a construir relaciones interpersonales con los lectores, oyentes y televidentes, parecen ser más útiles para organizaciones informativas más pequeñas. Allí, los contactos pueden ser más personales e íntimos, y el volumen de estos generalmente no es tan abrumador como para las organizaciones mediáticas más grandes.

\section{Los desafíos de capital y de ingresos}

Los desafíos gerenciales más críticos que afrontan hoy las empresas informativas involucran manejos de capital e ingresos. El capital (presupuesto) es el dinero fundacional mediante el cual las empresas son conformadas y continúan operando, mientras que los ingresos provienen dependiendo del modelo de negocio creado por la compañía.

La adquisición de capital a través del mercado de valores ${ }^{3}$ — una opción muy popular entre las grandes compañías mediáticas desde la década de los setenta y hasta el final del milenio - es cada vez menos viable, pues supone riesgos mayores para los potenciales inversionistas, especialmente cuando empresas informativas se encuentran involucradas. Compañías que cotizan en la Bolsa, dedicadas principalmente a la producción de contenidos informativos, presentan ahora significativas debilidades en comparación con los medios enfocados en proveer contenidos de entretenimiento,

3 La emisión de acciones en la bolsa de valores solía ser un instrumento común para la recaudación de fondos de capitales por parte de las grandes compañías mediáticas que operan en mercados desarrollados, especialmente en los Estados Unidos. Se destacan en este modelo las compañías mediáticas norteamericanas NBCUniversal, Disney, Time Warner, Viacom y News Corp. La japonesa SONY y la alemana Bertelsmann. Debido al tamaño limitado de los mercados y al propio alcance de las empresas, las acciones en bolsa por parte de compañías de medios en Latinoamérica no es una práctica conocida. En la región, los capitales de inicio y los presupuestos de operación provienen, en el caso de los medios comerciales, de inversionistas privados que crean compañías de medios bajo la figura de empresas limitadas o de sociedades anónimas. (N. del T.) 
u otros sectores económicos como los servicios de telecomunicaciones, la producción de medicamentos o los contratistas aeroespaciales o de defensa. Esto crea un ambiente en el cual los inversionistas presionan a las empresas informativas a generar retornos de la inversión a corto plazo, debido a que no esperan un crecimiento futuro del valor de sus activos.

Entre 2008 y 2009, un número importante de firmas de periódicos de gran reconocimiento en Estados Unidos, por ejemplo, padeció por la falta de recursos de capital. Varias compañías —entre ellas McClatchy Newspapers, Gannett Co. y New York Times Co- realizaron inversiones significativas en la compra de activos mediáticos mediante el préstamo de dinero, poniendo en riesgo el patrimonio de sus empresas. Cuando el valor de las acciones de las empresas se desplomó, dicho patrimonio disminuyó, elevando los costos sobre el capital prestado y generando crisis financiera. Varias compañías, particularmente Tribune Corp. y The Journal Register Co., fueron conducidas a la bancarrota porque acumularon una gran deuda debido a préstamos de capitales que no pudieron pagar.

El capital es también un asunto importante para la creación de compañías online, así como para pequeñas empresas mediáticas de alcance local e imprentas que tratan de reemplazar las funciones y el cubrimiento perdidos como consecuencia de la disminución de los recursos de las grandes organizaciones. Muy pocas son capaces de obtener capital de las fuentes de financiación tradicionales para poder pagar servicios, equipos, oficinas y salarios necesarios para garantizar operaciones efectivas. Muchas, en cambio, deben luchar financieramente y confiar en fondos inciertos proporcionados por fundaciones y benefactores. La experiencia ha demostrado, sin embargo, que tales fondos tienden a ser limitados y ofrecidos solamente por un corto periodo de tiempo, por tanto, estas operaciones de inicio enfrentan una presión significativa para generar ingresos por otros medios (Picard y van Weezel, 2008).

Desde el surgimiento de Internet como un medio viable hace cerca de una década y media, y la década desde cuando las comunicaciones móviles se hicieron posibles, las preocupaciones sobre la manera como los 
proveedores de información pueden generar ingresos se han mantenido vigentes, pues los modelos de ingresos efectivos para respaldar el acopio y la distribución de noticias siguen mostrando deficiencias. La publicidad online hasta ahora no ha logrado producir suficientes ingresos para sostener la actividad informativa y los usuarios han estado reacios a pagar por acceder a las noticias por Internet. Algunos dueños de sistemas de medios, liderados por Rupert Murdoch, planearon el cobro de noticias online proveídas por sus periódicos, pero la demanda de este servicio por parte del consumidor aún es incierta. Si el requerimiento de pago por la información reduce significativamente los niveles de lectura online, concurrentemente afectará los ingresos que son generados por la publicidad digital, por lo que se advierte un riesgo en esta estrategia.

Motivar al público a pagar por las noticias online no resulta tan simple como mover contenido a un servidor que requiere una suscripción de pago o un micropago para el uso individual. Obtener pagos a cambio de historias simples que también son cubiertas por otros cincuenta periodistas y que se encuentran disponibles sin ningún costo será frenar la demanda. El único modelo de pago por noticias que funcionará es aquel que provea un valor agregado que no pueda ser encontrado en ninguna otra parte. En consecuencia, las empresas informativas tendrán que poner más énfasis en la creación de valor para el consumidor.

Encontrar maneras de proporcionar el capital adecuado a las organizaciones de noticias, y que estas puedan generar ingresos, es esencial para encontrar la forma en que las industrias informativas puedan hacer frente a los efectos del entorno cambiante de los medios.

\section{Repensar y recrear las empresas informativas}

Los cambios referidos anteriormente al nuevo ambiente informativo nos obligan a confrontar cuestiones fundamentales sobre la importancia de las empresas informativas en la sociedad. Esto requiere una reflexión sobre qué funciones son necesarias para informar al público, para unir a la sociedad en su conjunto y para apoyar la participación democrática y el grado en el cual nuevas formas de comunicación y nuevos sistemas de medios sirven a 
estas funciones y alteran o reemplazan algunas de las funciones de los proveedores tradicionales de información.

También debemos reflexionar sobre si las actividades de los grandes medios, desarrolladas para para atender las necesidades de la sociedad de masas en los siglos XIX y XX, son la mejor manera para servir a la sociedad en el siglo XXI. Estas preguntas resultan inquietantes si uno es dueño, trabaja o quiere trabajar en una compañía de medios de comunicación. No obstante, analistas sociales e investigadores necesitan hacer frente a tales cuestiones si estamos llamados a encontrar soluciones para los retos actuales.

El hecho de que la financiación y la estructura de los medios informativos y periodísticos se haya convertido en objeto de gran interés público constituye un aspecto altamente positivo para la sociedad y para la industria informativa. La escala y el alcance otorgados hoy a estos asuntos son muy superiores a los experimentados durante varias generaciones. Proliferan los esfuerzos por crear y fundar nuevos tipos de organizaciones dedicadas al acopio y la difusión de información basadas en tecnologías contemporáneas - a menudo producto de la iniciativa de periodistas desempleadosy a pesar de los desafíos financieros algunas de ellas comienzan a tener una influencia significativa en la vida pública.

Sin embargo, no existen soluciones claras y simples a los retos que afrontan hoy las empresas informativas formalmente establecidas. Lo cierto es que la transformación seguirá ocurriendo. Estas empresas heredadas tendrán que encontrar caminos más efectivos para emplear sus recursos actuales y para explorar las oportunidades ofrecidas por otras formas de operar y de estructurar la labor periodística. Esto implica que tanto empresas como periodistas deban volverse más flexibles, más dispuestos a evolucionar, a explorar nuevas oportunidades y a adoptar una actitud más empresarial. Esto demanda cambios culturales significativos en las organizaciones y en las personas que trabajan para ellas.

Es probable que el futuro del periodismo y de las organizaciones informativas produzca mayor variedad de empresas de las que existen hoy, con una gama más amplia de estructuras de financiación y de soporte. Unas 
pocas empresas, tanto públicas como privadas, desempeñarán un importante rol en el ámbito informativo, acompañadas de una variedad de pequeñas compañías comerciales, o sin ánimo de lucro, creadas mediante figuras tipo fideicomiso y respaldadas económicamente por fundaciones. Las noticias locales online y los portales informativos probablemente surgirán como actores locales significativos, donde las condiciones económicas no permiten la publicación de periódicos o la transmisión de información local. Igualmente, se podrá desarrollar una variedad de organizaciones especializadas en tópicos y en temas específicos, además de agencias de intercambio de noticias.

El proceso de repensar, rediseñar y reformar las empresas informativas podrá resultar frustrante a veces, incluso desorientador y plagado de angustias. Pero jamás debería ser visto como la ruina para el periodismo o para las necesidades informativas de la sociedad. Si se infunden procesos con el propósito de crear efectividad y medios mejorados para acopiar y divulgar noticias e información, facilitando la discusión pública y comprometiendo al público en la participación democrática, conoceremos organizaciones y estructuras organizativas nuevas, y procesos que emergerán en el siglo XXI, que mejoren y respalden a las organizaciones ya existentes, faciliten la creación de otras nuevas e incrementen el acceso del público, el uso y la interacción con las noticias y la información.

\section{Referencias}

Albarran, A. (2005). Historical Trends and Patterns in Media Management Research En Albarran, A. B., Chan-Olmsted, S. and Wirth, M. O. (eds.). Handbook of Media Management and Economics (pp. 3-22). Mahwah: Lawrence Erlbaum.

Almqvist, K. and Thomas, I. (2008). The Future of Public Service Broadcasting. Stockholm: Axel and Margaret Ax - Johnson Foundation.

Bakardjieva, M. (2005). Internet Society: The Internet in Everyday Life. London: SAGE publications. 
Barnhurst, K. G. and Nerone, J. C. (2001). The Form of News: A History. New York: Guilford Press.

Becker, L. and Schonbach, K. (1999). Audience Response to Media Diversification. Mahwah: Lawrence Erlbaum.

Castells, M. (2001). The Internet Galaxy. Oxford: Oxford University Press.

Currah, A. (2009). What's Happening to Our News: An Investigation into the Likely Impact of the Digital Revolution of the Economics of News Publishing in the UK. Oxford: University of Oxford, Reuters Institute for the Study of Journalism.

European Audiovisual Observatory (2009). Statistical Yearbook. Strasbourg: European Audiovisual Observatory.

Fortune Global 5 oo (2009). Fortune, European edn, 20 July.

Fuchs, C. (2007). Internet and Society: Social Theory in the Information Age (Routledge Research in Information Technology and Society). London: Routledge.

Iosifidis, P., Steemers, J. and Wheeler, M. (2005). European Television Industries. London: British Film lnstitute.

Küng, L., Picard, R. G. and Towse, R. (eds.) (2008). The Internet and the Mass Media. London: SAGE Publications.

Murdoch, G. and Golding, P. (2001). Digital Possibilities, Market Realities: The Contradictions of Communications Convergence. Socialist Register, 38, 111-29.

Napoli, P. (2003). Audience Economics: Media Institutions on the American Marketplace. New York: Columbia University Press.

Nielsen Media Research (2003). Nielsen People Meter Sample. 
Nissen, C. S. (2006). Making a Difference: Public Service Broadcasting in the European Media Landscape. Eastleigh: John Libbey Publishing.

Office of National Statistics (2006). The Time Use Survey 2005, July. Available at http://www.statistics.gov.uk/cci/article. asp?id=1600.

Picard, R. G. (2002). The Economics and Financing of Media Companies. New York: Fordham University Press.

Picard, R. G. (2004). Environmental and Market Changes Driving Strategic Planning in Media Firms. En Picard, R. G. (ed.). Strategic Responses to Media Market Changes (pp. 1-17). Jonkoping: Jonkoping International Business School, Jonkoping University.

Picard, R. G. (2005a). Historical Trends and Patterns in Media Economics. En Albarran, A. B., Chan-Olmsted, S. and Wirth, M. (eds.). Handbook of Media Management and Economics (pp. 23-36). Mahwah: Lawrence Erlbaum.

Picard, R. G. (ed.) (2005b). Media Product Portfolios: Issues in Management of Multiple Products and Services. Mahwah: Lawrence Erlbaum.

Picard, R. G. (2006). Journalism, Value Creation, and the Future of News Organizations. Working Paper 2006-4. Cambridge: Joan Shorenstein Center for the Press, Politics, and Public Policy, John F. Kennedy School of Government, Harvard University.

Picard, R. G. and van Weezel, A. (2008). Capital and Control: Consequences of Different Forms of Newspaper Ownership. International Journal on Media Management, 10 (1), 22-31.

Pickard, V., Stearns, J. and Aaron, C. (2009). Saving the News: Toward a National Journalism Strategy. Washington DC: Free Press.

Price Waterhouse Coopers (2005). Global Entertainment and Media Outlet, 2005-2009. New York: PriceWaterhouseCoopers. 
Reilly Center for Media and Public Affairs (2008). The Breaux Symposium: New Models for News. Baton Rouge: Louisiana State University.

SIFO Research International (2008). Advertising Avoidance: The Quiet Consumer Revolt. Stockholm: SIFO Research lnternational.

Stuart, G. (2008). You Can't Avoid Ad Avoidance, Adweek. 6 October. Recuperado de http://www.adweek.com/aw/content_display/ community/columns/other-columns e3id9a975e26c8545c5ao2obbogo 8182476 .

University of California, Berkeley, School of lnformation Management and Systems (2004). Project: How Much lnformation 2003'. Recuperado de http://www.ischool.berkeley.edu/files/images/hmi2003.gif.

van Dijk, J. A. G. M. (2005). The Network Society: Social Aspects of New Media. London: SAGE Publications.

WARC (2008). World Advertising Trends 2008. Henley-on-Thames: World Advertising Research Center.

World Press Trends (annual). Paris: World Association of Newspapers. 